\title{
Magnetic resonance image evaluation of pallidotomy lesions: a volumetric and shape analysis
}

Jeffrey M. Burns, B.A., Steve Wilkinson, M.D., John Overman, B.S., Jennifer Kieltyka, B.A., Thorsten Lundsgaarde, B.A., Travis Tollefson, B.S., William C. Koller, M.D., Ph.D., Rajesh Pahwa, M.D., Alexander I. Tröster, Ph.D., Kelly E. Lyons, Ph.D., Solomon Batnitzky, M.D., Louis Wetzel, M.D., and Michael A. Gordon, Ph.D.

Imaging Resource Center and Departments of Surgery (Division of Neurosurgery), Neurology, Radiology, and Pharmacology, Toxicology, and Therapeutics, University of Kansas Medical Center, Kansas City, Kansas

Determination of acute pallidotomy-produced lesion volumes, pre- and postpallidotomy globus pallidus (GP) volumes, and assessment of lesion shape using magnetic resonance (MR) imaging-based computerized segmentation (contouring) and three-dimensional rendering was made in 19 patients. Magnetic resonance image slice thickness $(1.5 \mathrm{~mm}$ or $6 \mathrm{~mm})$ was not found to be a significant factor influencing contour-based pallidotomy lesion volume estimates. Previously reported lesion volumes produced by pallidotomy have often been estimated using the ellipsoid volume formula. Using 1.5-mm-thick MR sections, contour-based pallidotomy-produced lesion volumes were significantly different from those volumes estimated by the ellipsoid formula. Globus pallidus volumes, estimated by contouring $\mathrm{T}_{2}$-weighted MR images, were bilaterally similar $(2.4 \pm 0.37 \mathrm{ml}$ [right]; $2.2 \pm 0.45 \mathrm{ml}$ [left]). Postoperative GP volumes were found on the contralateral, unlesioned side to be $2 \pm 0.45 \mathrm{ml}$ and on the lesioned side to be $1.25 \pm 0.45 \mathrm{ml}$. Using the contralateral, unlesioned side as a reference volume, approximately $39 \pm 14 \%$ of the GP was visibly affected on the lesioned side. Seventeen of 18 patients had a favorable outcome with reduced dyskinesias and "off" time with improvement in parkinsonian symptoms.

Analysis of computerized three-dimensional rendering of pallidotomy-produced lesions based on MR images showed no relationship between lesioning technique and resulting lesion shape. Important factors in the volumetric analysis of pallidotomy lesions are identified and allow reasonable assessment of the pallidotomy lesion volume and shape and the extent of the affected GP.

Key Words * pallidotomy * radiofrequency lesions * Parkinson's disease * globus pallidus volume * magnetic resonance imaging * stereotaxy

Pallidotomy has reemerged as a surgical treatment option for Parkinson's disease. $[4,12]$ The rationale for pallidotomy is based on the need to reduce hyperactivity of the globus pallidus internus (GPi). The GPi hyperactivity is thought to result from both increased drive from subthalamic nuclei $[9,14]$ and decreased inhibitory tone due to the degenerating substantia nigra. Stereotactic radiofrequency (RF) lesioning in the 
GPi results in significant reductions in parkinsonian symptoms and drug-induced dyskinesia without significant morbidity and mortality.[9,12] As a basis for assessment of such RF lesions, precise description of lesion size and shape represents an important initial step.

Accordingly, this study uses both image analysis and computerized three-dimensional reconstruction techniques based on magnetic resonance (MR) imaging to measure volumes and assess pallidotomy-produced lesion shape. Previous investigators $[4,7,11,13]$ have reported RF lesion volumes produced by pallidotomy to range between $26 \mathrm{~mm}^{3}$ and $150 \mathrm{~mm}^{3}$. Some volume estimates have been based on assumptions concerning lesion shape (ellipsoid). Three-dimensional rendering of the lesion allows direct assessment and classification of lesion shapes, whereas image segmentation (contouring) approaches on a MR image-by-image basis may result in more precise lesion volume estimates. The relationship between volume and shape of the lesions produced by pallidotomy together with the specifics of the RF lesioning approach may be clarified by the present work.

\section{CLINICAL MATERIAL AND METHODS}

\section{Patient Population}

Between February 1996 and August 1996 MR imaging studies obtained in 19 patients with Parkinson's disease who had undergone stereotactic pallidal RF lesioning were analyzed at the University of Kansas Medical Center. These patients were selected for pallidotomy on the basis of the presence of drug-induced dyskinesias and motor fluctuations with severe "off" periods when they were not receiving medication. Exclusion criteria included dementia on neuropsychological profile, presence of significant white matter or deep gray matter lesions on computerized tomography (CT) scans, and serious medical illness. All patients were otherwise healthy and fit to withstand the stress of the surgery. The mean age of all patients was 64.8 years (range 47-75 years). There were eight men with a mean age of 62.6 years (range 47-75 years) and 11 women with a mean age of 66.9 years (range 62-74 years).

\section{Preoperative Planning}

Patients were generally in the functionally "off" state at the time of surgery as a result of either decreased dosages or absence of medication. Approximately 2 hours before surgery, a stereotactic frame (Cosman-Roberts-Wells Stereotactic Arc System; Radionics, Inc., Burlington, MA) was attached at four points on the patient's head following administration of a local anesthetic at attachment points.

Computerized tomography head scans were obtained with the gantry angled to approximate the angle of the anterior commissure-posterior commissure (AC-PC) plane. Computerized tomography scanning was performed using a high-speed scanner (Advantage System; General Electric Medical Systems, Milwaukee, WI) in nonhelical mode. Several scout images were obtained with a slice spacing of $10 \mathrm{~mm}$ to verify that the gantry was sufficiently angled to approximate the AC-PC plane and to determine the region for imaging the $\mathrm{AC}$ and the $\mathrm{PC}$. Images through the region of the $\mathrm{AC}-\mathrm{PC}$ plane were acquired at a $1-\mathrm{mm}$ slice thickness and a 48-cm field of view (FOV) with a 512 X 512 matrix. After selection of a slice containing both the $\mathrm{AC}$ and the $\mathrm{PC}$, an initial target site was determined using a target of $3 \mathrm{~mm}$ anterior to the midcommissural point, $20 \mathrm{~mm}$ lateral to the intercommissural line (ICL), and 3 to $6 \mathrm{~mm}$ ventral to the ICL. The target site's ventral position was adjusted according to individual anatomical differences depending on the location of the optic tract. If the optic tract was located in the CT images, that site was determined to be the final target.

\section{Surgical Procedure}


The patient was taken to the operating room where a local anesthetic was applied. A burr hole was made and a glass-tipped microelectrode of platinum-iridium wire with a shaft diameter of $0.66 \mathrm{~mm}$, an exposed recording tip length of 10 to $15 \mu$, and an initial impedance of 1 to $1.5 \mathrm{Mohm}$ (Atlantic Research Systems, Inc, Atlanta, GA) was inserted. The target was approached at an angle ranging between $45 \mathrm{~s}$ and $70 \check{\text { š to }}$ the AC-PC plane. Microelectrode field potential recordings were begun $20 \mathrm{~mm}$ above the proposed target using a microelectrode recording system (ARS NeuroSystem 2; Atlantic Research Systems, Inc.). The amplitude, frequency, and pattern of action potentials were recorded. The patient's arms and legs were passively moved to determine changes in neuronal firing patterns related to movement.

The microelectrode was inserted to the level of the optic tract, immediately ventral to the GP. Approximation to the optic tract was confirmed by recording a characteristic decrease in neuronal firing cellular activity over a distance of several millimeters. Once the decreased neuronal activity was noted, localization to the optic tract was confirmed by flashing a light in the patient's eye to elicit a response. If this response was heard, then microstimulation up to 50 mamps with a frequency of $125 \mathrm{~Hz}$ was used to produce phosphenes. Positive responses from these tests indicated the electrode was positioned in the optic tract.

Three to five tracts were made along the same trajectory and the cellular responses were recorded from $20 \mathrm{~mm}$ superior to the CT-determined target to the level of the optic tract. The cellular responses were then compared with a section from the Schaltenbrand and Bailey atlas[15] to determine electrode location in the basal ganglia.

Potential cellular targets were defined in several ways. Cellular recordings indicative of the GPi are characterized by high amplitude activity with fast and irregular frequency. An additional characteristic of potential target cells is a response to motor movement in the most affected extremity. If the patient presents with tremor, cells that fire synchronously with the tremor can also be found.[13] These cellular recording properties were used to define the optimum target for lesioning. Lesioning was not performed until the most dorsal and most ventral extents of the desired lesion were defined.

Once the target had been electrophysiologically defined, the microelectrode was replaced by a temperature-monitoring thermistor and lesion electrode that had either a 1.1-mm diameter and exposed 3-mm tip or a 1.6-mm diameter and 2-mm exposed tip (Radionics, Inc.). A lesion generator system (model RFG-3C; Radionics, Inc.) was used to generate the lesion.

\section{Lesion Creation}

Two general approaches were used in the formation of the entire lesion. One method was used to produce a cone-shaped lesion (RP Iacono, personal communication, 1996) and is referred to in this study as the graded approach. As the lesioning electrode was retracted in 2-mm increments, both temperature and duration were increased according to the following general protocol. Three to six discrete lesions were produced beginning at $75 \check{\mathrm{s} C}$ for 60 seconds and then increasing temperature to $80 \check{\text { š, }} 85 \check{s}$, and a maximum of $90 \check{s}$. The time spent producing the lesion was also increased from 60 seconds for the earliest discrete lesions to 120 seconds in the last one to two lesions placed in the tract. In one case, the duration of lesioning was not increased but held at a constant of 60 seconds for each discrete lesion placed.

The second method, referred to in this study as the constant approach, required maintenance of constant temperature (75ธ̌C) and duration (60 seconds) for the lesioning process while the electrode was retracted in $1-\mathrm{mm}$ or $2-\mathrm{mm}$ increments. Between three and five discrete lesions were placed within one or two 
tracts.

\section{Magnetic Resonance Imaging}

Magnetic resonance (MR) imaging of the head was obtained in each patient before and within 1 day following the surgical procedure. Magnetic resonance studies were performed using a 1.5-tesla system (model 63SPA; Siemens, Iselin, NJ) with a circularly polarized head coil. The following MR sequences were acquired: $\mathrm{T}_{1}$-weighted sagittal (TR, $500 \mathrm{msec}$; TE, $15 \mathrm{msec}$; FOV, $250 \mathrm{~mm}$; matrix, 224 X 256;

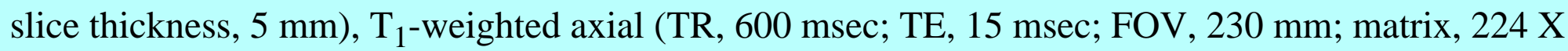
512; slice thickness, $6 \mathrm{~mm}$ ), $\mathrm{T}_{1}$-weighted coronal (TR, $600 \mathrm{msec}$; TE, $15 \mathrm{msec}$; FOV, $230 \mathrm{~mm}$; matrix,

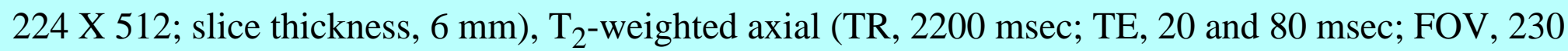
mm; matrix, 192 X 256; slice thickness, $6 \mathrm{~mm}$ ), three-dimensional (3-D) magnetization prepared rapid gradient echo (MPRAGE) acquisition (TR, $10 \mathrm{msec}$; TE, $4 \mathrm{msec}$; TI, $750 \mathrm{msec}$; flip angle 10; matrix, 192 X 256; slice thickness, 1.5-mm; FOV, $250 \mathrm{~mm}$ ). Magnetic resonance images were transferred by local area network to a computer system (model HP9000 J200; Hewlett-Packard, Palo Alto, CA) with a VISUALIZE 48 graphics subsystem. Analysis of MR images was performed using Omniview 2- and 3-D visualization software obtained from 3D Biomedical Imaging, Inc. (Shawnee Mission, KS).

The 3-D MPRAGE protocol was used to obtain axial images with a slice thickness of $1.5 \mathrm{~mm}$. This protocol offered excellent spatial resolution because of the thinness of the slice, which minimized volume averaging effects. The thin slice made it possible to maintain adequate resolution after multiplanar image reformations. Increased contrast of $\mathrm{T}_{2}$-weighted images compared with 3-D MPRAGE images offered increased resolution of anatomy such as the internal capsule, GP, and the putamen. Because these structures could be best visualized in the $T_{2}$-weighted images, they were used for segmenting (contouring) these structures.

\section{Data Segmentation}

Contouring or data segmentation is the outlining of anatomical boundaries in all images containing the structure. This process was used in determining volume, and it supports 3-D reconstruction and object rendering. Original 3-D MPRAGE MR images were acquired with a matrix of 192 X 256. The Omniview data entry program resamples the image data to create a $256 \times 256$ image, resulting in a pixel dimension of approximately $1 \mathrm{~mm}$. For data segmentation, the image undergoing contouring is enlarged to $600 \times 600$, with a resultant decrease in pixel dimension to approximately $0.4 \mathrm{~mm}$. Both manual tracing and thresholding approaches were used. Manual tracing is done by using a mouse to trace the structural boundaries. Thresholding is based on selection, on a gray scale level, of pixels within a region of interest (ROI) that defines the object. The ROI is determined by the user either by tracing or by adjustment of a deformable ellipse, encompassing the intended area to be contoured. The software generates a contour along the outer edge of the selected pixels within the ROI. Both methods may be applied on a slice-by-slice basis. Structures contoured using $\mathrm{T}_{2}$-weighted images by either of the above methods included inner and outer zones of the lesions and the GP, putamen, and internal capsule. We also used $\mathrm{T}_{1}$-weighted image sets for contouring of inner and outer lesions.

Both $\mathrm{T}_{1}$ - and $\mathrm{T}_{2}$-weighted images exhibit signals corresponding to pallidal lesions that are primarily composed of two zones with distinct boundaries. The lesion signals are composed of a distinct inner region surrounded by a distinct outer region. In $\mathrm{T}_{1}$-weighted images, these two zones are seen in each 
patient and consist of a hyperintense inner region surrounded by a hypointense outer region (Fig. 1 upper). Typically, $\mathrm{T}_{2}$-weighted images exhibited an inner, central zone of hypointensity and an outer zone of hyperintensity.

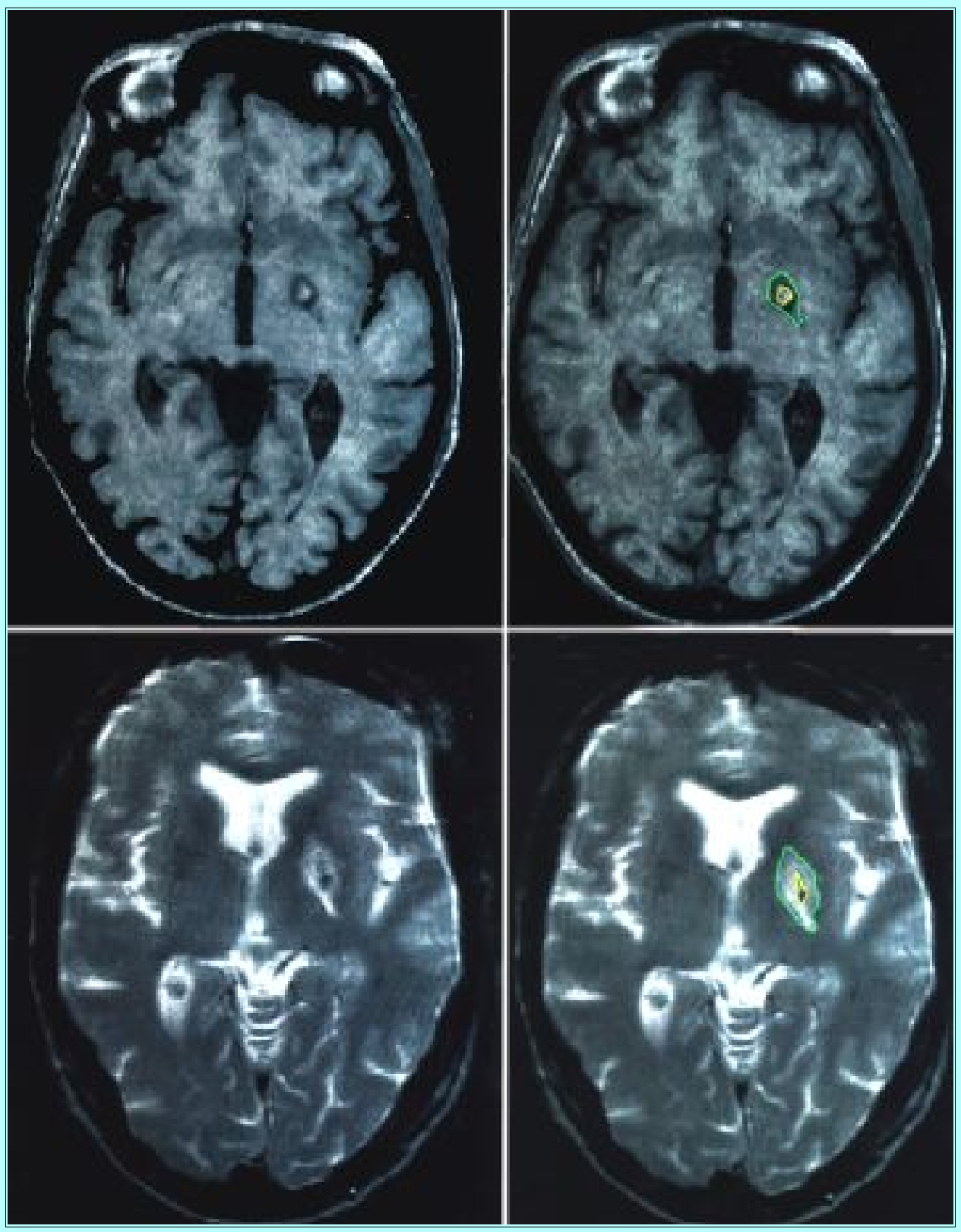

Fig. 1. Axial MR 3-D MPRAGE $\mathrm{T}_{1}$ - and $\mathrm{T}_{2}$-weighted images demonstrating zones of pallidotomy-produced lesion and contours. Upper left: The two zones of an acute pallidotomy-produced lesion are demonstrated on a 3-D MPRAGE $\mathrm{T}_{1}$-weighted image. The inner zone of relative hyperintensity represents an area of RF coagulation and is surrounded by an outer zone of hypointense signals, presumably edema. Upper right: The lesion signals have been segmented (contoured) by placing contours along the boundaries of the two zones. Every successive slice containing the lesion will be similarly contoured. Contours are used to calculate volumes and to produce 3-D renderings. Lower left: The two zones of an acute pallidotomy-produced lesion are demonstrated on a $\mathrm{T}_{2}$-weighted image with an echo time of $80 \mathrm{msec}$. In contrast to the $\mathrm{T}_{1}$-weighted image in Upper left and right, the inner zone is hypointense and the outer zone hyperintense. Lower right: The image shown in lower left with added contours. 
For inner and outer lesion volume estimations, intrarater variability in contouring was assessed using three trials conducted days apart to reduce bias. Before the next trial all previous contours were deleted. For volume estimations of GP, intrarater variability in contouring was also assessed using three trials conducted days apart. Before the next trial all previous contours were deleted.

\section{Combination of $T_{2}$-Weighted Data Sets}

Following image alignment, if required, data sets from $\mathrm{T}_{2}$-weighted TE 20 images and $\mathrm{T}_{2}$-weighted TE 80 images were combined. The fusion of the two image sets facilitated contouring of basal ganglia anatomy. Omniview data segmentation routines allow switching between multiple image sets. Because $\mathrm{T}_{2}$-weighted images acquired using an echo time of $80 \mathrm{msec}$ resulted in better visualization of the outer lesion and the GP and $\mathrm{T}_{2}$-weighted images acquired using an echo time of $20 \mathrm{msec}$ usually allowed good resolution of the internal capsule and the putamen, both image sets were used for data segmentation of basal ganglia structures. By combining the two image sets, the anatomy and the pallidal lesion were adequately visualized to allow contouring. These contours were used in volume determination and 3-D reconstructions.
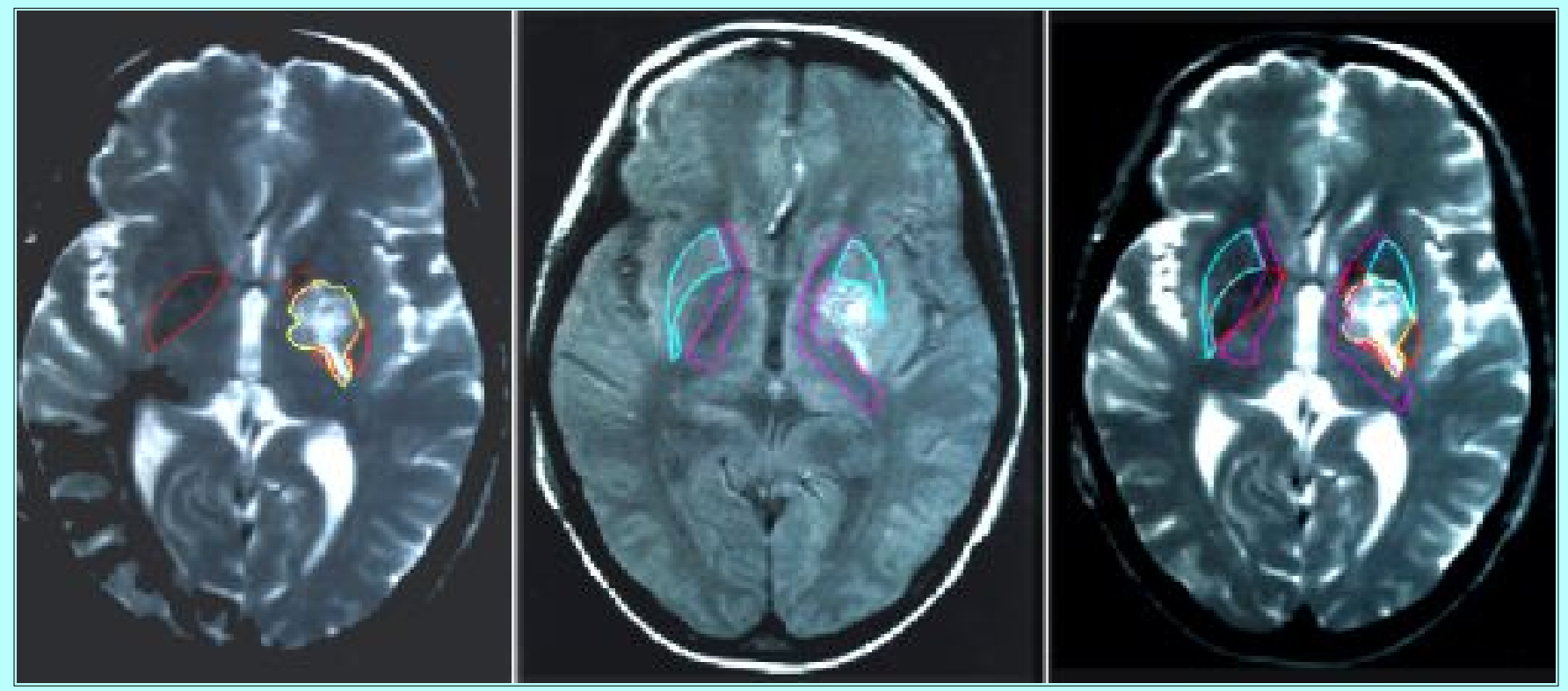

Fig. 2. Magnetic resonance $T_{2}$-weighted images demonstrating contouring of the lesion, GP, putamen, and internal capsule in combined $\mathrm{T}_{2}$ data. Left: $\mathrm{A}_{2}$-weighted image with an echo time of $80 \mathrm{msec}$ allows good resolution of the GP and the outer lesion. The images produced with this protocol were used to contour the GP (red) and the lesion (yellow). Center: The $\mathrm{T}_{2}$-weighted images with an echo time of $20 \mathrm{msec}$ were generally used for contouring of the internal capsule (magenta) and the putamen (blue). Right: Contours based on both image sets $\left(\mathrm{T}_{2}\right.$-weighted TE $80 \mathrm{msec}$ and $\mathrm{T}_{2}$-weighted TE $20 \mathrm{msec}$ ) were combined. This approach allowed both imaging protocols to be used in contouring the lesion and internal anatomy.

Fifteen $\mathrm{T}_{2}$-weighted image data sets were combined. Structures contoured include: contralateral unlesioned GP, lesioned GP, putamen bilaterally, internal capsule bilaterally, and the outer lesion. By toggling between either the TE 20 or TE 80 image data sets, structures were outlined. Only those areas of the lesion-side GP that were visible on the MR image and not residing within the outer boundary of the 
lesion were contoured (Fig. 2). Three-dimensional surface reconstruction based on these contours is shown in Fig. 3.

The contralateral, unlesioned GP volume was compared with the volume of the residual GP (lesion side). Subtracting the lesioned GP volume from the unaffected GP gives an estimate of the amount of the GP that is visibly affected by RF lesioning.
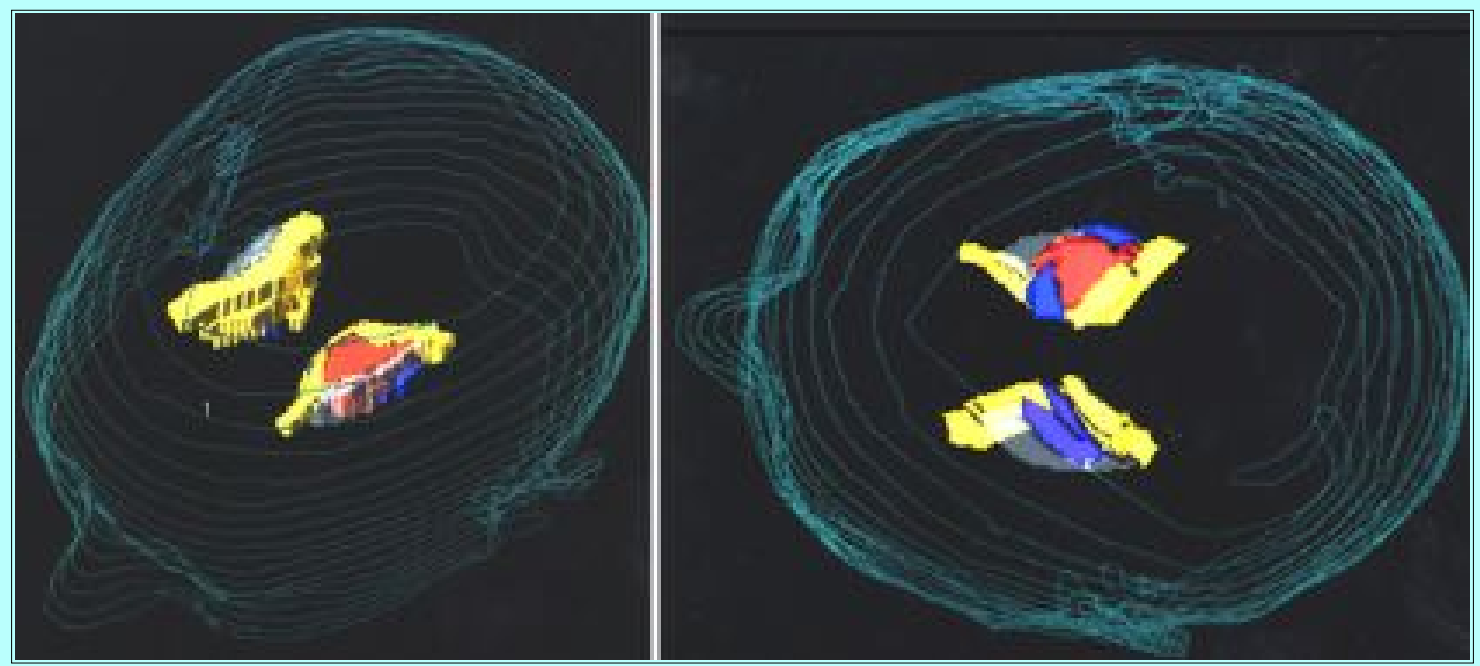

Fig. 3. Contours based on combined $\mathrm{T}_{2}$-weighted data (Fig. 2) have been used to produce a 3 -D reconstruction illustrating the relationship of the lesion and the internal anatomy. Left: Superior and anterolateral views of the internal anatomy and outer boundary of the lesion (red). Contours of the head are seen in blue with the patient's nose located in the lower left corner. The lesion is located in the patient's left GP and lies adjacent the internal capsule (yellow) and putamen (transparent white). Left: Posterior view of the internal anatomy and the outer boundary of the lesion (red). The patient's nose points toward the top of the figure. The lesion is located within the patient's left GP (blue) and has invaded the putamen (transparent white) beyond the margin of the GP. Note the contralateral and unlesioned anatomy. Internal capsule is represented in yellow.

\section{Three-Dimensional Reconstruction and Rendering}

The application software supported 3-D surface reconstruction of contoured structures. The GP, putamen, internal capsule, and the lesion were reconstructed and rendered in 3-D (Fig. 3). This approach allowed relationships of the lesion and surrounding anatomy to be appreciated. Inner and outer lesions were also reconstructed to allow shape characterization.

\section{Lesion Shape}

Lesion shape was characterized by inspection of the 3-D surface rendering of the lesions after contouring of 3-D MPRAGE MR images. The lesions were classified without prior knowledge of the specific lesioning protocol. Lesion shapes were categorized as conal, cylindrical, spherical, ellipsoidal, irregular, or combinations of these forms. The inner lesion zone and outer lesion zone were categorized separately. Cylindrical characterization is defined as maintenance of a stable lesion diameter along the full course of the lesion axis. Conal shape was defined as an increasing lesion diameter along the course of the lesion. Other lesions were characterized as spherical or ellipsoidal. Representative 3-D lesion shapes are illustrated in Fig 4. 

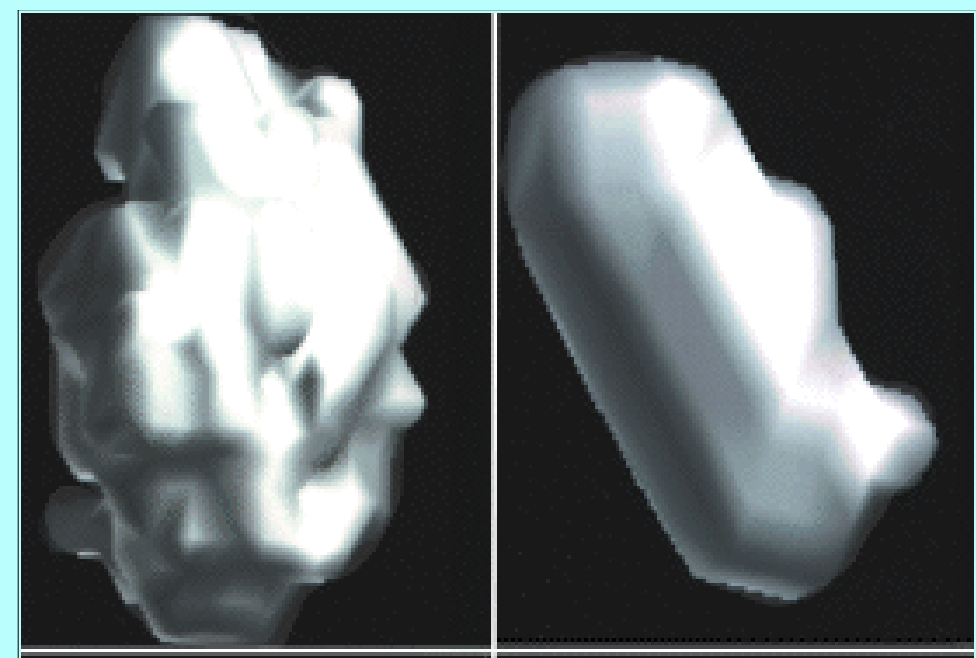

Fig. 4. Three-dimensional renderings of inner lesion contours (Fig. 1) demonstrating four lesion shapes. Volumes (below) are based on 3-D MPRAGE 1.5-mm-thick images. Upper left: Ellipsoidal inner lesion. Upper right: Cylindrical inner lesion. Lower left: Spherical inner lesion. Lower right: Conal inner lesion.

\section{Volumetric Analysis}

Lesion and GP volumes were computed using two methods. Method 1: Lesion and GP volumes were

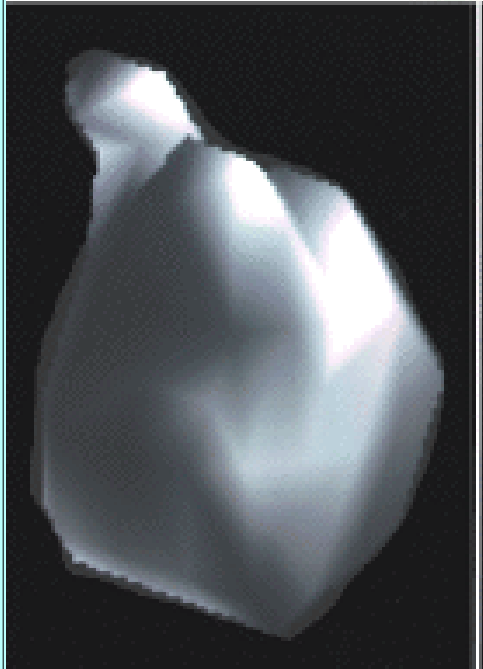

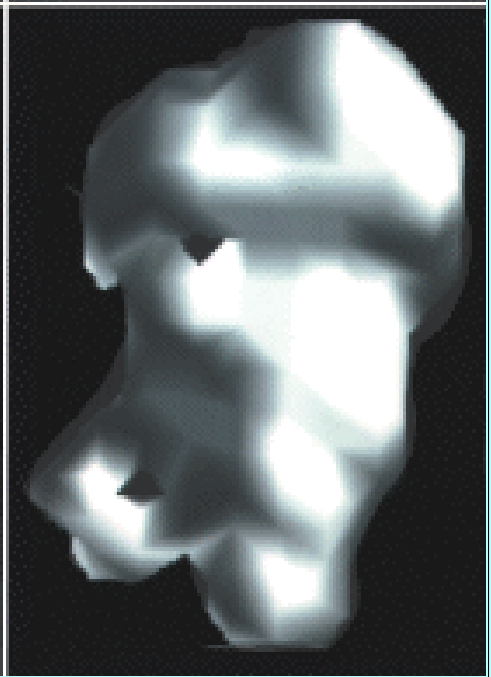
calculated by the application software directly from the contours. The application software computes volume based on determination of the area of each contoured region per slice multiplied by the slice thickness (Simpson's rule).

Method 2: Lesion volumes were also calculated by applying the ellipsoid volume formula ( $4 / 31 / 2(a b c)$. This method of volume determination has been used by previous investigators $[2,4,7,11]$ to estimate lesion volumes and is based on the assumption that the lesion is ellipsoidal. In our calculation using the ellipsoid formula, (4/3 1/2 (abc), a, b, and c represent one-half of the longest dimension of the lesion in each of the coronal, axial, and sagittal planes. The axial, sagittal, and coronal images containing the largest lesion diameter were identified and the longest dimensions were measured on the displayed image using the application software. Distance is computed following specification of the two points of interest using the mouse interface. Lesion volumes were calculated using this method for both 1.5-mm and 6-mm-thick MR images. To obtain the coronal and sagittal planes in 3-D MPRAGE 1.5-mm image sets, multiplanar reformations were performed. Computer-assisted multiplanar image reformations allow visualization of various MR image planes. For example, sets of sagittal MR images can be created from a set of original axial images. Because the 1.5-mm images were acquired only in the axial plane, coronal and sagittal MR image reformations were performed for estimation of lesion dimension in each orthogonal plane. The $\mathrm{T}_{1}$-weighted images (slice thickness of 6 $\mathrm{mm}$ ) were acquired in axial, coronal, and sagittal planes and therefore were used directly. However, $\mathrm{T}_{2}$-weighted image sets could not be used to determine volume using the ellipsoid formula because only axial images were available and significant loss of resolution would have occurred upon reformation.

\section{Statistical Analysis}

The mean and standard deviation (SD) of RF lesioning and GP volumes were determined. Lesion volumes obtained in the same patient using either different volume calculating methods or different MR imaging protocols were analyzed using Student's paired t-test. Because complete image data were not available in all patients, volume analysis was performed only on completely paired patient subsets for which complete image data were available. 
The effects of different lesioning techniques were analyzed with Student's unpaired t-test. Correlation coefficients were calculated to assist in identifying possible relationships between lesioning variables and inner and outer lesion volumes.

\section{Clinical Outcome}

The clinical results of pallidotomy were assessed 1 to 3 months postoperatively by a neurologist who used patient history, clinical rating of symptoms and signs, and estimates in quantities of dyskinesias and times at which the patient was not receiving medication, as measured by the Unified Parkinson's Disease Rating Scale (UPDRS).

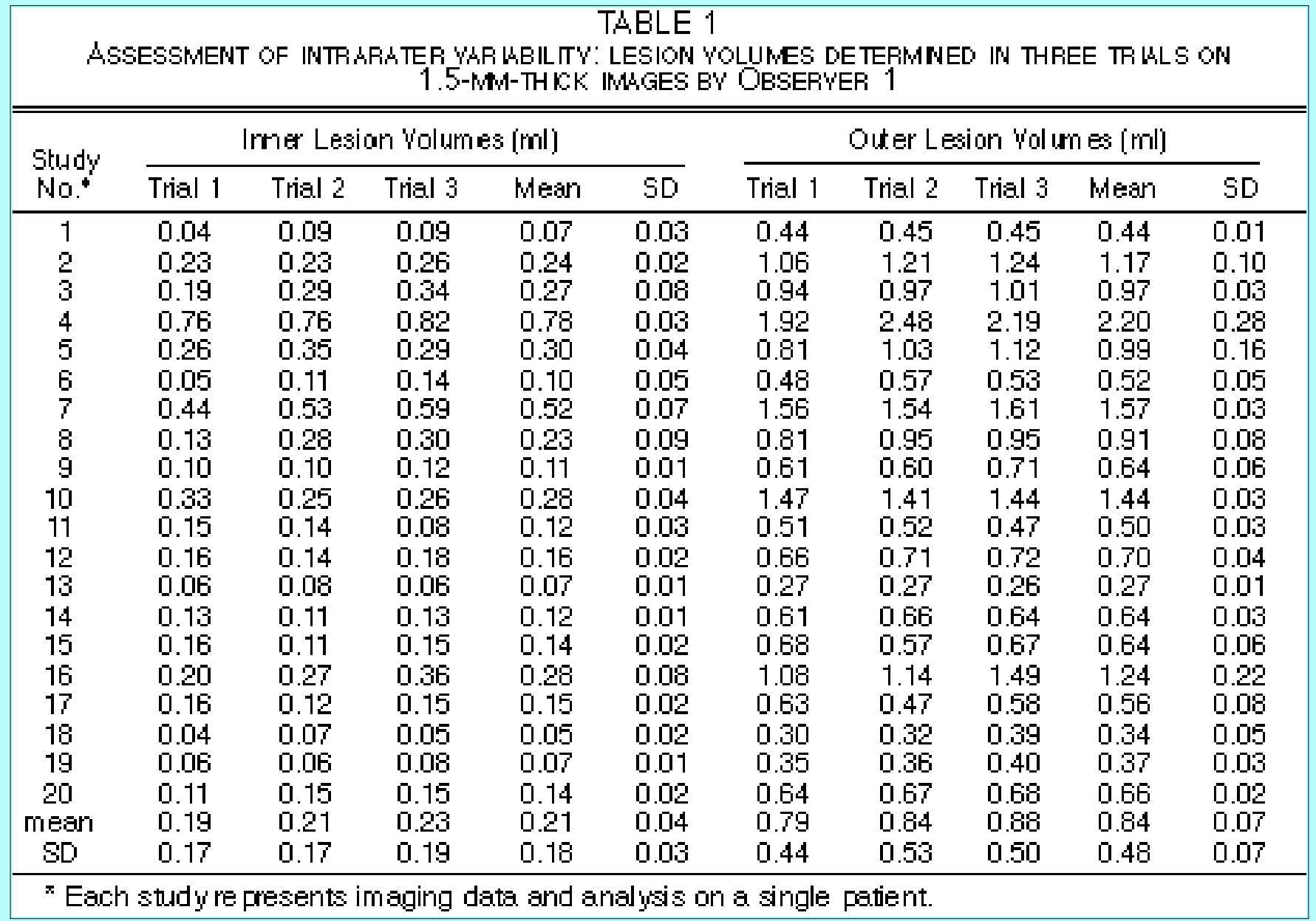

\section{RESULTS}

\section{Intrarater Volume Determinations}

Table 1 shows an example of multiple volume determinations by contouring performed by the same observer using 3-D MPRAGE images. Multiple trials were conducted using a total of four observers to assess the intrarater and interrater reliability of volume determination. A mean and SD were determined for the three independently determined volume estimates on each individual lesion. For the first observer, the mean of all inner lesion volumes was approximately $0.20 \mathrm{ml}$, with an uncertainty of approximately $0.18 \mathrm{ml}$. The mean of all outer lesions was $0.8 \mathrm{ml}$, with an uncertainty of $0.5 \mathrm{ml}$. Three additional observers independently measured both inner and outer lesions using the same 3-D MPRAGE image data. The second observer found a mean of all inner lesion volumes of approximately $0.12 \mathrm{ml}$, with an uncertainty of approximately $0.07 \mathrm{ml}$ and a mean of all outer lesion volumes of approximately $0.65 \mathrm{ml}$, with an uncertainty of $0.4 \mathrm{ml}$. The third observer found a mean of all inner lesion volumes of 
approximately $0.20 \mathrm{ml}$, with an uncertainty of approximately $0.13 \mathrm{ml}$ and a mean of all outer lesion volumes of approximately $0.69 \mathrm{ml}$, with an uncertainty of $0.4 \mathrm{ml}$. The fourth observer found a mean of all inner lesion volumes of approximately $0.22 \mathrm{ml}$, with an uncertainty of approximately $0.16 \mathrm{ml}$ and a mean of all outer lesion volumes of approximately $0.79 \mathrm{ml}$, with an uncertainty of $0.44 \mathrm{ml}$. Observers 1 , 3 and 4 all had previous experience in contouring pallidotomy lesions and used similar rules for distinguishing lesion margins. Observer 2 had no previous experience in contouring pallidotomy lesions and was not instructed in advance concerning definition of lesion boundaries. Interrater differences are relatively small for lesion volume estimates derived from contours drawn by Observers 1, 2, and 4 . However, volumes determined by Observer 2 are generally smaller for inner lesion volume estimates.

\section{Effect of Slice Thickness on Volume Estimations}

The effects of MR slice thickness on lesion volumes were also determined by contouring. Both 1.5-mm and 6-mm image sets were obtained in 17 patients. Inner lesion volumes based on 6-mm-thick MR image sets (in 17 patients) resulted in a mean of $0.18 \pm 0.13 \mathrm{ml}(\mathrm{SD})$. The 1.5 -mm-thick images resulted in a mean inner volume of $0.21 \pm 0.15 \mathrm{ml}(\mathrm{SD})$. Student's paired t-test showed no significant difference in the estimated volumes derived from $6-\mathrm{mm}$ and $1.5-\mathrm{mm}$ MR data $(\mathrm{p}=0.195)$. The mean outer lesion volume based on 6-mm MR image sets (17 patients) resulted in a mean of $0.85 \pm 0.50 \mathrm{ml}$ compared with outer lesion volumes for 1.5 -mm-thick images of $0.89 \pm 0.48 \mathrm{ml}$. Student's paired t-test did not show a significant difference in estimated volumes derived from 6-mm and 1.5-mm MR data ( $\mathrm{p}=0.094)$; (Fig. $5)$.

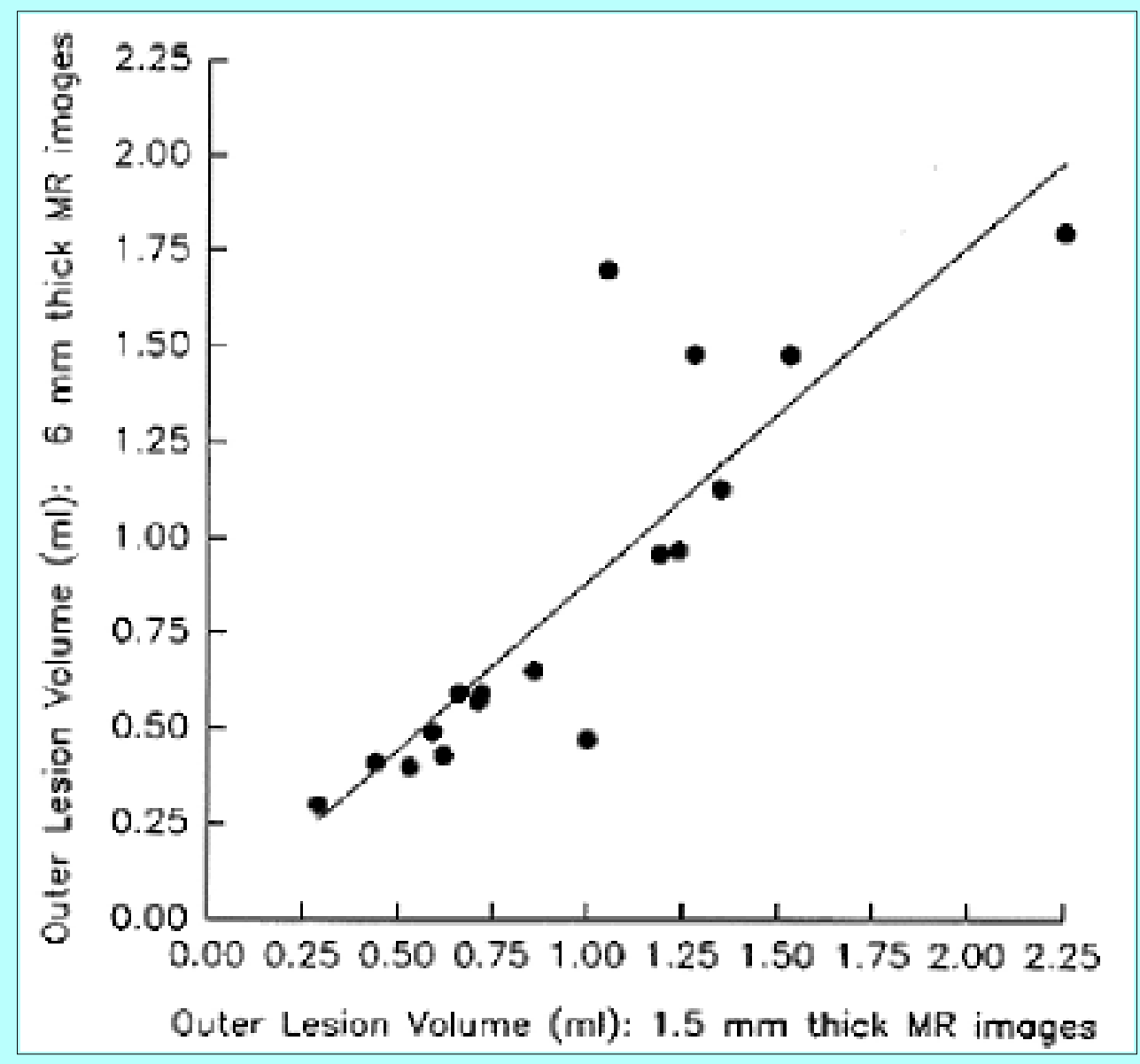

Fig. 5. Plot illustrating the relationship between outer lesion volumes estimated from 1.5-mm- and 6-mm-thick MR images. 


\section{Relationship Between Inner and Outer Lesion Volumes}

Figure 6 illustrates the relationship between inner and total lesion volumes as determined by contouring 3-D MPRAGE images. The lesion volumes are well correlated; their correlation coefficient is greater than 0.88 .

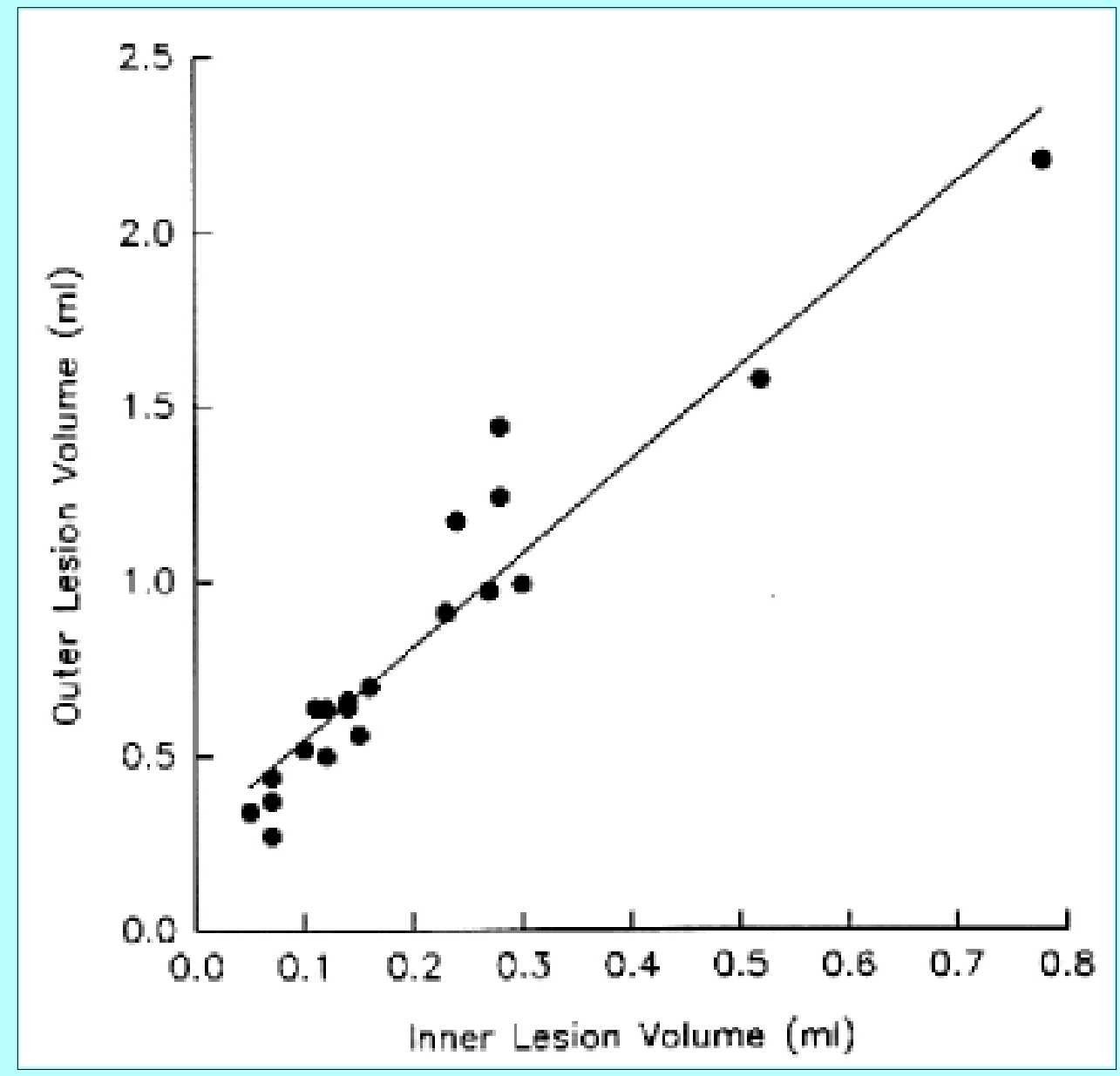

Fig. 6. Representative plot illustrating the relationship between inner and total (outer) lesion volume. Data presented are the mean of three trials by Observer 1 (Table 1).

\section{Ellipsoid Formula Volume Estimates Compared With Contour-Based Estimations}

Lesion volume estimates using the ellipsoid formula were compared with those derived from contours for both 1.5-mm and 6-mm image sets. The same 15 1.5-mm- and 6-mm-thick MR image sets were used for both ellipsoid and contour-based volume determinations. Volumes calculated using the ellipsoid formula resulted in larger means and SDs compared with volumes of the same lesions determined by contouring in all cases.

For $1.5-\mathrm{mm}$ image sets, inner lesion volumes calculated from contours averaged $0.22 \pm 0.16 \mathrm{ml}$, which was significantly different $(\mathrm{p}<0.03)$ from that obtained using the ellipsoid formula, $0.39 \pm 0.39 \mathrm{ml}$. For 1.5 -mm image sets, outer lesion volumes calculated from contours $(0.96 \pm 0.50 \mathrm{ml})$ were also significantly different $(\mathrm{p}<0.001)$ from that obtained using the ellipsoid formula $(1.34 \pm 0.74 \mathrm{ml})$. Statistically significant differences between 6-mm image sets were not observed. 
Lesion volumes based on either $\mathrm{T}_{1}$ - or $\mathrm{T}_{2}$-weighted 6-mm MR image data were also determined. There was no significant difference between inner lesion volume estimates using either $T_{1}$ or $T_{2}$ sequences in 16 patients $(\mathrm{p}=0.13)$. However, outer lesion volumes determined from $\mathrm{T}_{2}$-weighted TE 80 images in 17 patients resulted in a mean outer lesion volume of $2.88 \pm 1.51 \mathrm{ml}$ which was significantly different $(\mathrm{p}<$ $0.001)$ than outer lesion volume estimates $(0.85 \pm 0.5 \mathrm{ml})$, based on $\mathrm{T}_{1}$-weighted axial images.

\section{Globus Pallidum Volumes}

Estimates of GP volumes were determined from seven preoperatively acquired $\mathrm{T}_{2}$-weighted TE 80 data sets using the contouring method. The GP volumes were determined to have a mean of $2.4 \pm 0.37 \mathrm{ml}$ (eight patients) on the right side and $2.2 \pm 0.45 \mathrm{ml}$ (eight patients) on the left. Intrarater variability in estimating GP volume was approximately $20 \%$.

Postoperative GP volumes were found using combined $\mathrm{T}_{2}$-weighted TE 20 and TE 80 data sets. Contralateral, unlesioned GP volumes were determined to have a mean of $2.00 \mathrm{ml}$ with an SD of $0.45 \mathrm{ml}$. The remaining GP on the lesioned side had a mean of $1.25 \mathrm{ml}$ with an SD of $0.45 \mathrm{ml}$. The difference between the unlesioned GP volume and the lesioned GP volume represents a rough estimate of the amount of GP seen to be affected on MR imaging by the placement of the lesion. This affected volume includes both the inner core and surrounding edema. The mean of the difference is $0.75 \mathrm{ml}$ with an SD of $0.22 \mathrm{ml}$. The ratio of this difference to the contralateral, unlesioned GP gives an estimate of the percentage of the initial, intact GP that has been visibly affected. The mean of this percentage is $39 \%$ with an SD of $14 \%$ and a range of 23 to $75 \%$.

\section{Effect of Lesioning Process Variables on Lesion Shape}

Graded lesioning produced three cylindrical and three ellipsoidal inner lesions with only one resembling a cone. Five ellipsoidal outer lesions were produced using the graded lesioning method. The constant lesioning method produced five ellipsoidal and five cylindrical inner lesions and two spherical lesions. No cone-shaped inner lesions were produced using the constant method. However, two outer lesions produced with the constant method contained some conal characteristics. It is clear that the shape is variable and not consistently ellipsoidal. The graded temperature and duration approach, intended to produce a cone-shaped lesion, failed to do so for both inner and outer lesions in six of the seven attempts. In only one inner lesion (Fig. 4 lower left) and two outer lesions did it slightly resemble a cone. A second observer produced similar lesion shape classification for inner lesions; however, significant variation in shape classification between observers was noted for the outer lesions.

\section{Clinical Outcome}

Seventeen of eighteen patients reported a favorable outcome after surgery. These patients had a greater than $50 \%$ reduction in dyskinesias and a greater than $25 \%$ reduction in "off" time. Parkinsonian signs consisting of bradykinesia and tremor were reduced. One patient had no change in clinical symptoms after pallidotomy.

\section{DISCUSSION}

\section{Magnetic Resonance Signal Intensity From Lesions}

Magnetic resonance signal intensities corresponding to the inner lesion zone are thought to represent a 
hemorrhagic coagulative necrotic volume indicative of permanent changes due to RF heating. $[2,6,16]$ By contrast, the outer zone exhibits MR characteristics most consistent with edema[2,6,16] and regresses with time.[16] The two lesion zones contoured using $T_{1}$ - and $T_{2}$-weighted $M R$ data in this study are similar to previous descriptions.[16] These two lesion zones were noted on nearly all image sets used in the present study and were taken as measures of a central necrotic core volume and surrounding edema.

Precise assessment of pallidotomy lesion volumes is an important step in understanding the role of pallidotomy in the treatment of Parkinson's disease. Therefore, an appreciation of the methods for volume determination and factors that introduce error is necessary. One approach involves the use of the ellipsoid formula.[4,7,11,13] Another approach uses slice-by-slice contours of the lesion as a basis for volume estimation. Use of the ellipsoid formula takes only the largest lesion dimension in the coronal, axial, and sagittal planes to estimate a volume based on the assumption that the lesion is ellipsoidal. However, contour-based estimation of volume makes no assumptions about lesion shape. Moreover, analysis of lesion shapes based on 3-D surface reconstructions (Fig. 4) suggests that lesions are not consistently ellipsoidal. Estimation of lesion volume by contouring may thus give a more accurate representation.

\section{Variability in Lesion Volume Estimates}

Several factors involved in contouring introduce error into the estimation of lesion volume. Subjectivity is involved in the determination of a boundary for contouring. A lesion boundary may be indistinct so that precise determination of the boundary is difficult. This factor is probably responsible for most of the variability in intrarater trials, even for experienced observers. This problem seemed more serious when low contrast between lesion and surrounding tissue occurred. To reduce this variability it is important to form a set of rules prior to contouring so that consistency in boundary determination can be maintained as much as possible throughout the trial. For instance, in this study, the outer boundary was defined as the first perceptible gray scale changes in the area of the lesion.

Another consideration that generally predisposes to variability in lesion volumes within a patient group is that constant lesion volume between patients is not an objective of the pallidotomy procedure. Because the lesion location, number of discrete lesions at a given temperature, and duration of lesioning (decided on kinesthetic and field potential estimates of pallidal neuronal activity) are individualized for each patient, lesion volumes would be expected to vary between patients.

\section{Effect of Slice Thickness on Volume Determinations}

Slice thickness showed no statistically significant effect on the estimation of volumes by contouring. Contouring using thinner MR image sections $(1.5 \mathrm{~mm})$ was expected to produce volume estimates that would be more accurate than those obtained from thicker sections $(6 \mathrm{~mm})$. Other factors, perhaps relating to the contouring process itself and volume averaging effects, may have been more significant than the number of contours that define the volume.

\section{$T_{1-}$ and $T_{2}$-Weighted Images}

Outer lesion volume estimates based on $\mathrm{T}_{2}$-weighted data were significantly higher in value than on $\mathrm{T}_{1}$-weighted data of the same slice thickness $(6 \mathrm{~mm})$. This result confirms earlier findings[2] that the largest lesion volumes were found in $\mathrm{T}_{2}$-weighted data (using the ellipsoid formula). In this study mean 
lesion volumes were on the order of three and a half times greater than $\mathrm{T}_{1}$-weighted data. As outer lesion volumes most likely correspond to edema, $[2,6,16]$ increased outer volumes determined with $\mathrm{T}_{2}$-weighted data most likely represent the increased sensitivity of $\mathrm{T}_{2}$-weighted MR imaging to edema.[1,10]

\section{Globus Pallidum Volumes}

Globus pallidum volumes estimated in this study are consistent with the volumes reported in the literature.[5,8] Globus pallidum volumes have been determined anatomically to be $2.04 \pm 0.39 \mathrm{ml}$ for the left side and $2.08 \pm 0.38 \mathrm{ml}$ for the right.[8] Estimates from triplicate determinations on preoperative GP volumes as well as the contralateral (nonlesion) GP volumes in this study fall within these ranges. In another study using MR images of 5-mm slice thickness, the GP volumes were estimated to be $1.61 \pm$ $0.45 \mathrm{ml}$ in the left and $1.87 \pm 0.42 \mathrm{ml}$ in the right.[5] In the present study, 1.5-mm images did not offer the contrast of $\mathrm{T}_{2}$-weighted 6-mm images and therefore the GP could not be consistently contoured using the $1.5-\mathrm{mm}$ data sets. However, in one set of $1.5-\mathrm{mm}$-thick images the contrast and resolution were exceptional and provided adequate views of the GP, allowing it to be contoured. In this one example, the volume was determined to be $1.9 \mathrm{ml}$, which agrees closely with our 6-mm slice-based determinations and determinations by other investigators.

Radiofrequency lesions affected approximately $40 \%$ of the GP (range 23-75\%). In determining these values, it was assumed that GP volumes are bilaterally equivalent. Very similar left and right pallidal volumes reported here $(2.2 \pm 0.4 \mathrm{ml}$ [left] to $2.4 \pm 0.4 \mathrm{ml}$ [right]) are consistent with previously reported volumes $[5,8]$ that show close to equivalent bilateral volumes. The percentages of GP affected by lesioning gave an estimate of the largest amount of GP affected. The estimate was generated using $\mathrm{T}_{2}$-weighted data, which produced a significantly larger total lesion volume than that found in the $\mathrm{T}_{1}$ data. However, estimates of inner lesion volumes based on $\mathrm{T}_{1}$ and $\mathrm{T}_{2}$-weighted $\mathrm{MR}$ image sets were similar. Therefore, inner lesion volume, based on $\mathrm{T}_{2}$-weighted TE 80 scans, was approximately $10 \%$ of the outer lesion volume. Accordingly, if most of the long-term therapeutic benefit is due to the inner core lesion, a percentage much less than $40 \%$ of affected GP may be responsible for the clinical efficacy of pallidotomy.

\section{Radiofrequency Lesioning Technique and Lesion Shape}

The graded temperature and duration approach was used in an effort to produce a cone-shaped lesion, but this approach failed in all but a single case. Lesioning approaches used in this study were not predictive of lesion shape. In a feline model, increased duration of current application tended to result in a lesion that was more cylindrical rather than spherical.[3] This result does not appear applicable to human pallidotomy using the particular graded or constant lesioning approaches applied in this study. The varying lesion shapes that may be seen in other pallidotomy studies may be the result of different lesioning approaches used (such as, number of tracts and discrete lesion number, temperature, or duration).[17]

In conclusion, volumetric analysis of pallidotomy-produced lesions combined with three-dimensional reconstruction and rendering allows reasonable assessment of the pallidotomy lesion volume, shape, and the extent of the GP affected. Postoperative MR-based characterization of pallidotomy-produced lesion volume and shape may allow better assessment of those lesioning factors that are important in producing optimal lesion sizes and shapes. Ultimately, correlation of lesion volumes and shapes with lesion location and clinical results may lead to a better understanding of the role of pallidotomy in the treatment of 


\section{Acknowledgment}

The authors are grateful for the expert assistance of the technical support staff of the Department of Radiology.

\section{Appendix}

Tables containing all data for inner and outer pallidotomy lesion volume estimates for each of four observers as well as methodological detail concerning electrophysiological refinement are available directly from the authors.

\section{References}

1. Bederson JB, Bartkowski HM, Moon K, et al: Nuclear magnetic resonance imaging and spectroscopy in experimental brain edema in a rat model. J Neurosurg 64:795-802, 1986

2. De Salles AA, Brekhus SD, De Souza EC, et al: Early postoperative appearance of radiofrequency lesions on magnetic resonance imaging. Neurosurgery 36:932-937, 1995

3. Dieckmann G, Gabriel E, Hassler R: Size, form and structural peculiarities of experimental brain lesions obtained by thermocontrolled radiofrequency. Confin Neurol 26:134-142, 1965

4. Dogali M, Fazzini E, Kolodny E, et al: Stereotactic ventral pallidotomy for Parkinson's disease. Neurology 45:753-761, 1995

5. Elkashef AM, Buchanan RW, Gellad F, et al: Basal ganglia pathology in schizophrenia and tardive dyskinesia: an MRI quantitative study. Am J Psychiatry 151:752-755, 1994

6. Farahani K, Mischel PS, Black KL, et al: Hyperacute thermal lesions: MR imaging evaluation of development in the brain. Radiology 196:517-520, 1995

7. Hariz MI: Correlation between clinical outcome and size and site of the lesion in computed tomography guided thalamotomy and pallidotomy. Stereotact Funct Neurosurg 54/55:172-185, 1990

8. Heckers S, Heinsen H, Heinsen Y, et al: Cortex, white matter, and basal ganglia in schizophrenia: a volumetric postmortem study. Biol Psychiatry 29:556-566, 1991

9. Iacono RP, Shima F, Lonser RR, et al: The results, indications, and physiology of posteroventral pallidotomy for patients with Parkinson's disease. Neurosurgery 36:1118-1127, 1995

10. Kamman RL, Go KG, Brouwer W, et al: Nuclear magnetic resonance relaxation in experimental brain edema: effects of water concentration, protein concentration, and temperature. Magn Reson Med 6:265-274, 1988

11. Laitinen LV: Pallidotomy for Parkinson's disease. Neurosurg Clin North Am 6:105-112, 1995

12. Laitinen LV, Bergenheim AT, Hariz MI: Leksell's posteroventral pallidotomy in the treatment of 
Parkinson's disease. J Neurosurg 76:53-61, 1992

13. Lozano A, Hutchison W, Kiss Z, et al: Methods for microelectrode-guided posteroventral pallidotomy. J Neurosurg 84:194-202, 1996

14. Lozano AM, Lang AE, Galvez-Jimenez N, et al: Effect of GPi pallidotomy on motor function in Parkinson's disease. Lancet 346:1383-1387, 1995

\section{Schaltenbrand G, Bailey P: Introduction to Stereotaxis, With an Atlas of the Human Brain.} Stuttgart: Thieme, 1959

16. Tomlinson FH, Jack CR Jr, Kelly PJ: Sequential magnetic resonance imaging following stereotactic radiofrequency ventralis lateralis thalamotomy. J Neurosurg 74:579-584, 1991

17. Tsementzis SA, Gillingham FJ, Gordon A, et al: Decerebrate rigidity produced in cats by focal stereotactic radiofrequency lesions. Acta Neurochir 59:13-33, 1981

Manuscript received November 1, 1996.

Accepted in final form January 17, 1997.

Address reprint requests to: Michael A. Gordon, Ph.D., Department of Pharmacology, Toxicology and Therapeutics, University of Kansas Medical Center, 39th and Rainbow, Kansas City, Kansas 66103. email: mgordon@kumc.edu 